\title{
Estrategias que modulan el síndrome de Burnout en enfermeros (as): una revisión bibliográfica
}

ISSN 1794-9831

E-ISSN 2322-7028

Vol. 14 No. 1

Ene - Jun 2017

Cúcuta, Colombia

Recibido:

27 de Mayo de 2016

Aprobado:

2 de Noviembre

de 2016

\section{RESUMEN}

Introducción: el síndrome de Burnout es la sensación de agotamiento y pérdida de interés por el trabajo que surge en profesionales que mantienen contacto diario con otras personas. Metodología: se realizó una revisión de artículos que reportan estrategias para modular el Burnout en profesionales de enfermería, en las siguientes bases de datos: Scielo, ProQuest, Sciencie Direct, Ovid Nursing, EBSCO, Medline, Pubmed y Wiley Online Library. Los descriptores en español, inglés y portugués utilizados fueron: Burnout, enfermeras, intervenciones. Se encontraron 1.833 artículos, de los cuales 17 cumplieron con los criterios de selección. Resultados: el efecto de las intervenciones pre y post para el Burnout fue medido con el Maslach Burnout Inventory y la Escala de Depresión y Ansiedad Hospitalaria, entre otros. Las intervenciones más reportadas fueron apoyo social, psico-educación, entrenamiento en la solución de problemas y en habilidades de comunicación. 16 artículos reportaron disminución o ausencia del Burnout después de las intervenciones, con cambios en las variables propuestas por Maslach y Jackson o en la sostenibilidad en el tiempo. Conclusión: se reportan estrategias para el Burnout sin distinción de las variables del mismo; sin embargo, se evidencian diferencias en los resultados frente a cambios positivos en cansancio emocional, en comparación con las otras variables.

PALABRAS CLAVE: adaptación psicológica, agotamiento profesional, enfermeros, despersonalización ${ }^{* * * *}$.

Para citar este artículo / Para citar este artigo/ To reference this article /

Vargas-Cruz LD, Niño-Cardozo CL, Acosta-Maldonado YY. Estrategias que modulan el síndrome de Burnout en enfermeros (as): una revisión bibliográfica. Rev. cienc. cuidad. 2017; 14(1): 111-131.

* Enfermera.

Magíster en Enfermería con Énfasis en Gerencia de

Servicios de Salud. Docente. Programa de Enfermería.

Universidad de

Ciencias Aplicadas y Ambientales

(U.D.C.A). Bogotá

D.C., Colombia.

Correo electrónico: luvargas@udca.

$\underline{\text { edu.co }}$

** Enfermera.

Especialista en

Cuidado Crítico,

Magíster en

Bioética Docente.

Programa de Enfermería. Universidad de Ciencias Aplicadas y Ambientales (U.D.C.A). Bogotá D.C., Colombia.

Correo electrónico: cnino@udca.edu.co

***Enfermera.

Empresa Social del Estado Sub Red Sur Hospital de Usme. Bogotá D.C.; Colombia. Correo electrónico: jeacosta@udca. edu.co

**** Descriptores en Ciencias de la Salud (DeCS), en la página http:// decs.bvs.br/E/ homepagee.htm de la Biblioteca Virtual en Salud del proyecto BIREME, de la Organización Mundial de la Salud y de la Organización Panamericana de la Salud. 
ISSN 1794-9831

E-ISSN 2322-7028

Vol. 14 No. 1

Ene - Jun 2017

Cúcuta, Colombia

\section{Strategies that modulate the Burnout syndrome in nurses: a bibliographic review}

\section{ABSTRACT}

Introduction: the Burnout syndrome is the feeling of exhaustion and loss of interest in the work that emerges in professionals that maintain daily contact with other people. Methodology: A review of articles that report strategies to modulate the Burnout in nursing professionals was performed in the following databases: Scielo, ProQuest, ScienceDirect, Ovid Nursing, EBSCO, Medline, Pubmed and Wiley Online Library. The descriptors used in Spanish, English and Portuguese were: Burnout, nurses, interventions. 1833 articles were found, in which 17 met the criteria of selection. Results: the effects of pre and post interventions for the Burnout was measured with the Maslach Burnout Inventory and the Hospital Anxiety and Depression Scale, among others. The interventions most reported were social support, psychoeducation, problem solving training and communication skills. 16 articles reported the decrease or absence of Burnout after the interventions, with changes in the variables proposed by Maslach and Jackson or in the sustainability over time. Conclusion: strategies for the Burnout are reported without distinction of the variables; however, differences are evidenced on the results regarding positive changes in emotional tiredness, compared to other variables

PALABRAS CLAVE: adaptation psychological, Burnout professional, nurses, depersonalization. 


\section{Estratégias que modulam a síndrome de Burnout em enfermeiros (as): uma revisão de literatura}

\section{RESUMO}

Introdução: a síndrome de Burnout é um transtorno adaptativo crônico associado às demandas e exigências laborais, cujo desenvolvimento é insidioso e frequentemente não reconhecido pelo indivíduo, que gera cansaço emocional, sensação de esgotamento e perda de interesse pelo trabalho, principalmente apresentando-se em professionais que mantem contato diário com outras pessoas. Metodologia: realizou-se uma revisão de artigos que reportam estratégias para modular o Burnout em professionais de enfermagem, nas seguintes bases de dados: Scielo, ProQuest, Sciencie Direct, Ovid Nursing, EBSCO, Medline, Pubmed e Wiley Online Library. Os descritores utilizados em espanhol, inglês e português foram: Burnout, enfermeiras, intervenções. Encontraram-se 1.833 artigos, dos quais 17 cumpriram com os critérios de seleção. Resultados: o efeito das intervenções pré e pós para o Burnout foi medido com o Maslach Burnout Inventory e a Escala de Depressão e Ansiedade Hospitalar, entre outros. As intervenções mais reportadas foram apoio social, psico-educação, treinamento na solução de problemas e em habilidades de comunicação. 16 artigos reportaram diminuição ou ausência do Burnout depois das intervenções, com mudanças nas variáveis propostas por Maslach e Jackson ou na sustentabilidade no tempo. Conclusão: se reportam estratégias para o Burnout sim distinção das variáveis do mesmo; porém, se evidenciam diferenças nos resultados frente a mudanças positivas em cansaço emocional, em comparação com as outras variáveis.

PALAVRAS-CHAVE: adaptação psicológica, esgotamento professional, enfermeiras e enfermeiros, despersonalização. 
ISSN 1794-9831

E-ISSN 2322-7028

Vol. 14 No. 1

Ene - Jun 2017

Cúcuta, Colombia

\section{INTRODUCCIÓN}

El síndrome de Burnout (SB) se define como la sensación de agotamiento, decepción y pérdida de interés por la actividad laboral (1). Surge especialmente en aquellos que se dedican a profesiones de servicio, como consecuencia del contacto diario con otras personas en su trabajo (2), especialmente los profesionales de la salud y la educación, quienes son los colectivos del sector público más afectados por el SB (3), que lleva consigo problemas de salud mental (ansiedad, depresión, entre otros) (1).

Según Quiceno et al. (4), "el SB es una respuesta al estrés laboral crónico, acompañado de una experiencia subjetiva de sentimientos, cogniciones y actitudes que provocan alteraciones psicofisiológicas en la persona y consecuencias negativas para las instituciones laborales". "No es un proceso asociado a la fatiga sino a la desmotivación emocional y cognitiva que sigue al abandono de intereses que, en un determinado momento, fueron importantes para el sujeto" (4).

El SB repercute sobre la sociedad en general y es considerado un problema social y de salud pública debido a que genera aumento de los costos económicos y sociales (5), afecta la moral, el bienestar psicológico, físico y mental de los trabajadores, la calidad y el tratamiento que se les proporciona a los pacientes, además del funcionamiento administrativo de los servicios sanitarios, con sus consecuentes implicaciones como ausentismo, rotación, disminución de la productividad y de la calidad (56 ), aumento en los errores, lo cual repercute en la seguridad y salud del paciente (7) y bajos niveles de satisfacción del cliente (8).

Maslach y Jackson (2) resaltan tres características principales para el SB: el cansancio emocional o emotional exhaustion (EE, por sus siglas en inglés), la despersonalización (DP) y la falta de realización personal (PA). El primero se describe como un sentimiento de vacío (9) y "agotamiento ante las exigencias de la tarea por la que no se siente atractivo alguno" (10), puesto que crea relaciones emocionalmente independientes de los pacientes y reduce los encuentros con ellos al mínimo requerido, fenómeno que puede convertirse en indiferencia y falta de interés por las necesidades del otro (9).
El segundo, la despersonalización (DP), supone una conducta de rechazo hacia los pacientes (10): el individuo presenta reacciones negativas hacia ellos, se niega a ser cortés, denigra e incluso desprecia al paciente. Los sentimientos que pueden surgir son el cinismo y el desapego (9).

El tercero, la falta de realización personal (PA), según Tuesca et al. (10) consiste "en una actitud negativa hacia uno mismo y hacia el trabajo, pérdida de interés por éste, irritabilidad, baja productividad y pobre autoestima"; el individuo siente un fracaso profesional y el colapso de su autoestima puede llevar a la depresión (10).

Albaladejo et al. (11) y Chacón y Grau (12) señalan que el síndrome de Burnout afecta más a aquellas profesiones que requieren un contacto directo con las personas y con una filosofía humanística del trabajo, es decir, aquellas que necesitan altas dosis de entrega: enfermeros, médicos, trabajadores sociales, profesores y policías. De las anteriores, la profesión que hace más vulnerable a las personas para adquirir el SB es enfermería, por condiciones laborales como largas jornadas de trabajo, turnos nocturnos, sobrecarga, recursos insuficientes (13-14), condiciones laborales precarias, pérdida de seguridad laboral, bajos salarios, falta de recurso humano, necesidad de trabajar en más instituciones, insatisfacción laboral e incremento de demanda por responsabilidad civil (15).

Así mismo, la escasa autonomía para la toma de decisiones, la falta de reconocimiento profesional, el contacto cotidiano con el dolor, la enfermedad y la muerte, además de la falta de recompensa intrínseca y de retroalimentación positiva (13), condicionan el riesgo para el SB en la profesión de enfermería.

Las principales variables desencadenantes y facilitadoras del SB reportadas por la literatura se relacionan con factores personales, profesionales e institucionales, entre estos: la utilización de recursos técnicos en el trabajo, la ambigüedad y el conflicto de rol, el clima laboral, los contenidos del puesto de trabajo, las características de personalidad, la motivación laboral y el sentido de coherencia $(6,11-12)$.

Los factores protectores para prevenir el SB pueden ser personales u organizacionales. Dentro de los personales están: percepción de autoeficacia, 
autoestima y autoconfianza, sentimiento de competencia laboral y manejo de técnicas de counselling (de afrontamiento) (14-15). Para Edwards y Burnard (16), la autoestima es uno de los factores más importantes como moderador del estrés.

En cuanto a los factores organizacionales se encuentran: la cohesión y el apoyo grupal, la relación de confianza mutua, la realización de reuniones interdisciplinarias periódicas, la evaluación del grado de satisfacción del equipo de salud, la planificación de condiciones y la delimitación de las funciones y tareas de cada miembro del equipo (14-15).

El Consejo Internacional de Enfermeras (CIE), desde 1957, señala como funciones de enfermería: la promoción de la salud, la prevención de las enfermedades y el cuidado de enfermos físicos, mentales e impedidos, de todas las edades, en todos los sitios en los que se brinda atención de salud y en otros servicios comunitarios (17). De forma similar, en Colombia, estas competencias del profesional de enfermería están descritas en la Ley 266 de 1996 (18) que, además, incluye el desarrollo, diseño, implementación y control de políticas, proyectos de atención en salud y la dirección de servicios de salud de enfermería, instituciones y programas de atención primaria en salud.

Además, la multiplicidad de funciones dentro de la asistencia que realiza la enfermera (o), conlleva la realización de labores administrativas, educativas, investigativas y de docencia (13) que, en ocasiones, se conjugan en un mismo escenarios laboral e incrementan así el riesgo de padecer el SB.

Se evidencian responsabilidades amplias para el profesional de enfermería. En Colombia, la reforma al Sistema General de Seguridad Social en Salud (SGSSS) generó cambios en la lógica económica del sistema, llevándolo a un modelo de intervención privada $\mathrm{y}$, en el caso particular de enfermería, a la creación de cooperativismo asociado como mecanismo de flexibilidad laboral. Esta situación supone una desigualdad en el trabajo, precariza las condiciones laborales y trae consigo el estrés ocupacional y el SB (19).

Se han llevado a cabo estudios (20) dirigidos a la detección del SB en profesionales de enfermería.
Aiken et al. (21) indagaron por la situación laboral de 43.000 enfermeras en cinco países (Estados Unidos, Canadá, Inglaterra, Escocia y Alemania), de las cuales un $40 \%$ presenta EE en el Maslach Burnout Inventory (MBI), instrumento utilizado para medir estrés laboral o Burnout. También encontró que las condiciones laborales de las enfermeras son predictores importantes de la variación en los resultados del paciente.

En Hispanoamérica, el estudio de Grau et al. (22), realizado con 11.530 profesionales de la salud, encontró una prevalencia de SB de 14,9\% en España, $14,4 \%$ en Argentina, $7,9 \%$ en Uruguay y entre 2,5 y $5,9 \%$ en México, Ecuador, Perú, Colombia, Guatemala y El Salvador. Enfermería tuvo una prevalencia del 7,2\%.

En Chile, Melita etal.(20) realizaron una investigación sobre SB en enfermeras y encontraron dos grupos de profesionales que presentaban mayor riesgo para $\mathrm{EE}$ : aquellos con menos experiencia laboral y aquellos que tenían entre 50 y 59 años. En la escala del MBI, el nivel más elevado fue para PA, con un $40 \%$.

Álvarez et al. (23) encontraron que la edad predominante del SB está entre 20 y 29 años, en mujeres y con el EE y la DP. Al contrario, en Melita et al. (20) y Grisales et al. (24), la prevalencia más alta fue en hombres, fenómeno asociado a sentimientos de baja PA en el trabajo, ya sea por falta de apoyo social o bajo nivel de realización profesional. En otros países se encontró que el desgaste profesional puede afectar entre 14 y $63,3 \%$ del personal de enfermería (25).

En Colombia, Ávila et al. (26) evaluaron las variables demográficas y laborales del SB y encontraron mayor incidencia del SB en los servicios de urgencias y hospitalización. La dimensión con mayor puntuación fue la DP con $60,6 \%$, seguida por EE con $45,3 \%$ y baja PA en 31,3\%. En una investigación realizada en Cartagena (25), en enfermeras (os) del servicio de urgencias, se encontró una prevalencia del $26,6 \%$ del $\mathrm{SB}$, asociado con la insatisfacción laboral y con edad menor de 40 años. En Ibagué (24), la prevalencia fue de $20,1 \%$ y el $52,3 \%$ está en riesgo de padecerlo.

Las áreas donde el SB tiene mayor incidencia son: urgencias, unidad de cuidados intensivos (UCI), cuidados paliativos, hematología y oncología; la

(n)


ISSN 1794-9831

E-ISSN 2322-7028

Vol. 14 No. 1

Ene - Jun 2017

Cúcuta, Colombia enfermera (o) enfrenta a diario el sufrimiento humano con pacientes terminales y la inminencia de la muerte. En esta profesión se requiere de una madurez y experiencia clínica profesional que permita hacer frente a la toma de decisiones difíciles (11).

A pesar del gran número de estudios que investigan el $\mathrm{SB}$, pocos se encargan de reportar las intervenciones y son escasos aquellos que evalúan la eficacia de éstas (27), específicamente en el área de enfermería. La literatura (27) ha reportado dos tipos principales de intervenciones para el SB: las diseñadas para ayudar al individuo a hacer frente a los factores de estrés en el lugar de trabajo - que parte del supuesto de que el SB es el resultado de una mala adaptación de la persona a su lugar de trabajo-, y las intervenciones centradas en realizar cambios de los factores organizacionales.

Popp (28) menciona que las intervenciones centradas en el individuo incluyen las estrategias de afrontamiento. El afrontamiento o coping hace referencia a la manera como las personas responden a determinadas situaciones, distinguiéndose tres vertientes: el modo de afrontamiento dirigido al problema, el que va dirigido a la emoción y el que se orienta hacia la evaluación.

El afrontamiento dirigido al problema busca definirlo y encontrar alternativas de solución. Incluye las estrategias de requerimiento de información o asesoramiento, ejecución de acciones para resolver el problema con el desarrollo de planes alternativos y el desarrollo de gratificaciones o recompensas que generen satisfacción (28). El uso de modos de afrontamiento centrados en el problema trae mayor satisfacción laboral, menor cansancio emocional y, por lo tanto, menores niveles del Burnout (28).

Por su parte, el afrontamiento dirigido a la emoción pretende regular la respuesta emocional a través de la aceptación de la situación y de la imposibilidad de cambiarla, el control afectivo y la descarga emocional (28).

Para Popp (28), el afrontamiento centrado en la evaluación da significado a la situación, sus causas y soluciones mediante estrategias como el análisis lógico, la redefinición cognitiva positiva de los acontecimientos o la evitación cognitiva a través del olvido de la situación y la focalización en deseos y fantasías.
El meta-análisis de Laurentiu et al. (27) muestra otras intervenciones a nivel individual: las basadas en técnicas cognitivo conductuales (TCC), relajación, meditación, habilidades interpersonales y conocimiento; las TCC son eficientes para el control del estrés en el trabajo. Las intervenciones basadas en técnicas de relajación y meditación son utilizadas con mayor frecuencia para el control del estrés, con resultados mixtos para el Burnout (27).

Según Laurentiu et al. (27), las intervenciones dirigidas al desarrollo de habilidades interpersonales se basan en la generación de un clima de trabajo positivo y de grupos de apoyo. Si bien estas intervenciones aumentan la auto-eficacia, no tienen efecto significativo en la reducción o prevención del Burnout. Las intervenciones centradas en el desarrollo de conocimientos y habilidades relacionadas con el trabajo se basan en el supuesto de que los empleados mejoran el manejo del estrés mediante la utilización de sus nuevas capacidades (27).

En relación con las intervenciones para la prevención o reducción del SB en enfermeros (as), Edwards y Burnard (16) identificaron seis estudios llevados a cabo en el Reino Unido, uno en los Países Bajos y otro en los EE.UU, donde las intervenciones se han centrado en el individuo.

En la actualidad resulta necesario identificar las estrategias que modulan el SB en los profesionales de enfermería, ya que la calidad de vida laboral y el estado de salud físico y mental tienen repercusiones importantes a nivel personal e institucional (ausentismo, recambio de personal, disminución de la productividad y calidad del trabajo, entre otros) (14).

\section{METODOLOGÍA}

Se realizó una revisión sistemática de literatura entre enero de 2015 y junio de 2016, sobre las estrategias y acciones para intervenir en el profesional de enfermería con SB en las variables de cansancio emocional (EE), realización personal (PA) y despersonalización (DP). Como criterios de selección se establecieron los siguientes: artículos publicados entre 2000 y 2016, en idioma inglés y español, que permitieran acceso a texto completo y que reportaran intervenciones para el SB en profesionales de enfermería. 
Se realizó la búsqueda en las bases de datos de Scielo, Proquest, Sciencie Direct, Ovid Nursing, EBSCO, Medline, Pudmed y Wiley Online Library y repositorio de la Biblioteca Virtual en Salud (BVS). Se utilizaron como descriptores: strategies to modulate SB, interventions SB in nurses, interventions emotional exhaustion in nurse, depersonalization in nursing interventions, personal accomplishment interventions nursing.

La revisión de literatura comprendió la lectura de 1.833 resúmenes, de los cuales 345 corresponden al descriptor strategies to modulate sb síndrome/ estrategias para modular en sb, 283 para interventions $\mathrm{sb}$ syndrome in nurses / intervenciones para el sb en enfermería, 548 para interventions emotional exhaustion in nurse / intervenciones para cansancio emocional en enfermeras, 309 para depersonalization in nursing intervention/ intervenciones en despersonalización en enfermeras, y 348 para personal accomplishment interventions nurse / intervenciones personal en enfermería. Se seleccionaron 17 artículos que cumplieron con los criterios de inclusión, de los cuales 14 son investigaciones y 3 revisiones sistemáticas de la literatura. Los resultados se sistematizaron a través de fichas RAE (resumen analítico del escrito).

El procedimiento utilizado para la revisión fue: primero, búsqueda bibliográfica en diferentes bases de datos sobre el SB; segundo, selección de los artículos o referencias que cumplían con los criterios de inclusión; tercero, realización de fichas RAE para cada artículo; cuarto, clasificación de los resultados; quinto, redacción de los resultados más relevantes, y sexto, confrontación y verificación de los resultados con diferentes estudios realizados anteriormente.

\section{Medición pre y pos intervenciones}

Doce artículos corresponden al idioma inglés y cinco al español. El reporte de artículos por país fue: dos para EEUU, Turquía, Reino Unido y Noruega, uno para Colombia, Francia, Brasil, Italia, Chile, Canadá, Noruega, España y Holanda.

Los años de publicación fueron: cuatro para 2012, dos para 2010, uno para 2015, 2014, 2011, 2009, 2007, 2004, 2003 y 2002. En relación con las áreas donde se desarrollaron los estudios reportados por los artículos, cuatro corresponden al servicio de oncología, dos a unidades de cuidado intensivo, dos a servicios de hospitalización, dos a salud mental, uno a cuidado paliativo y dos no reportan área.

Cinco artículos se dirigen a personal sanitario y doce artículos a enfermeras (os). El número de participantes varía entre 9 y 248 enfermeras (os). Trece artículos se ubican en el paradigma cuantitativo; de estos, cuatro ensayos aleatorios, dos cuasi experimental, uno descriptivo, casos y controles, longitudinal mono céntrico, mixto (cualitativo, cuantitativo), observacional y no probabilístico.

Las evaluaciones pre y post intervención fueron medidas a través diversos instrumentos que se presentan en la Tabla 1. Se evidencia que el instrumento más utilizado corresponde al Maslach SB Inventory (MBI), creado en 1981 por Maslach y Jackson (2), siendo el instrumento clásico de medición más utilizado a nivel mundial para evaluar la presencia del $\mathrm{SB}$, independiente de las características de la muestra $\mathrm{y}$ de su origen (13).

Actualmente existen tres versiones de esteinstrumento: MBI-Human Services Survey (MBI-HSS), dirigido a los profesionales de la salud y que es la versión clásica; el MBI-Educators Survey (MBI-ES), orientado a profesionales de la educación, y el MBI-General Survey (MBI-GS), no exclusivo para profesionales, que mantiene la estructura tridimensional, contiene 16 ítems en total y denomina las dimensiones baja eficacia profesional, agotamiento y cinismo (13).

En el MBI, las puntuaciones de cada sub-escala se evalúan por separado, la relación entre las tres dimensiones no se muestra con una puntuación total y se calculan para cada individuo tres diferentes puntuaciones (29).

Los datos sociodemográficos se lograron mediante encuestas realizadas por los autores. Algunos estudios incluyeron variables que pueden afectar el desgaste como edad, experiencia laboral, nivel de educación y número de turnos noche (29).
ISSN 1794-9831

E-ISSN 2322-7028

Vol. 14 No. 1

Ene - Jun 2017

Cúcuta, Colombia 
Estrategias que modulan el síndrome de Burnout en enfermeros (as): una revisión bibliográfica • Luz Dalila Vargas-Cruz,Carmen Lucía Niño-Cardozo,Jessica Yurlay Acosta-Maldonado

ISSN 1794-9831

E-ISSN 2322-7028 Vol. 14 No. 1

Ene - Jun 2017

Cúcuta, Colombia
Tabla 1. Instrumentos utilizados para medición de impacto de estrategias en relación al SB.

\begin{tabular}{|c|c|c|}
\hline Instrumento & $\begin{array}{l}\text { Estudio que utilizó el } \\
\text { instrumentó }\end{array}$ & Descripción \\
\hline $\begin{array}{l}\text { Maslach SB } \\
\text { Inventory (MBI) }\end{array}$ & $\begin{array}{l}\text { Contreras et al. (13), Kravits } \\
\text { et al. (8), Isaksson et al. (30), } \\
\text { Goodman y Schorling, (31 ), } \\
\text { Edmonds et al. (7), Günusën y } \\
\text { Üstün, (29), Sabanciogullari y } \\
\text { Dogan (32), Ewers et al. (33), } \\
\text { Vásquez et al. (15), Italia et al. } \\
\text { (9), Le Blanc et al. (34), Quenot } \\
\text { et al. (35), Rodrigues et al. (36) }\end{array}$ & $\begin{array}{l}\text { El MBI es un cuestionario de } 22 \text { ítem, considerada como la herramienta de oro para el } \\
\text { SB, tiene tres escalas: EE, D y PA. }\end{array}$ \\
\hline $\begin{array}{lr}\text { Cuestionario } & \text { de } \\
\text { Afrontamiento } & \text { al } \\
\text { Estrés [CAE] } & \end{array}$ & Contreras et al. (13). & $\begin{array}{l}\text { "Escala de } 42 \text { ítem desarrollada y validada por Sandín y Chorot. El instrumento } \\
\text { fue diseñado para evaluar siete estilos básicos de afrontamiento: 1) focalizado en la } \\
\text { solución del problema, 2) auto focalización negativa, 3) reevaluación positiva, 4) } \\
\text { expresión emocional abierta, 5) evitación, 6) búsqueda de apoyo social, y 7) religión. } \\
\text { Tiene adecuadas propiedades psicométricas, con coeficiente promedio de fiabilidad de } \\
\text { Cronbach de } 0,79 \text { para las siete subescalas" (13). }\end{array}$ \\
\hline 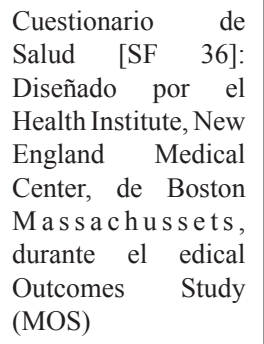 & Contreras et al. (13) & $\begin{array}{l}\text { "El SF36 es un instrumento psicométricamente sólido de } 36 \text { ítems, que evalúa la calidad } \\
\text { de vida a través de distintas dimensiones: función física (limitaciones físicas), rol físico } \\
\text { (interferencia en el trabajo y actividades diarias), dolor corporal (intensidad del dolor y } \\
\text { su efecto en las actividades), salud general (valoración personal de la salud), vitalidad } \\
\text { (sentimiento de energía), función social (interferencia en la vida social habitual), } \\
\text { rol emocional (interferencia en el trabajo u otras actividades diarias), salud mental } \\
\text { (depresión, ansiedad, control emocional y de la conducta), y evolución declarada de la } \\
\text { salud (valoración de la salud actual comparada con la de un año atrás)" (13). }\end{array}$ \\
\hline $\begin{array}{l}\text { Escala de Depresión } \\
\text { y Ansiedad } \\
\text { Hospitalaria } \\
\text { (HADS) } \\
\text { desarrollada por } \\
\text { Zigmond y Snaith }\end{array}$ & $\begin{array}{l}\text { Vásquez et al. (15), Rodrigues } \\
\text { et al. (36) }\end{array}$ & $\begin{array}{l}\text { Esta escala con adaptación española, ha sido diseñada para detectar síntomas de depresión } \\
\text { y ansiedad durante la semana anterior a la evaluación en población no psiquiátrica. } \\
\text { Con sus dos subescalas, proporciona una puntuación de ansiedad (HAD-A) y otra de } \\
\text { depresión (HAD-D) (15). }\end{array}$ \\
\hline $\begin{array}{l}\text { Job Stress Scale } \\
\text { (JSS) }\end{array}$ & Rodrigues et al. (36) & $\begin{array}{l}\text { Evalúa el estrés a partir de tres dimensiones: exigencias psicológicas, control y apoyo } \\
\text { social. }\end{array}$ \\
\hline $\begin{array}{l}\text { C u e st i o n a r i o } \\
\text { General de Salud } \\
\text { [General Health } \\
\text { Questionnaire] }\end{array}$ & Edmonds et al. (7) & $\begin{array}{l}\text { Este instrumento evalúa la enfermedad psicológica, se compone de } 12 \text { preguntas } \\
\text { relacionadas con el estrés, tales como depresión, capacidad para dormir, sentimientos de } \\
\text { competencia y capacidad para disfrutar de todos los días de la vida. }\end{array}$ \\
\hline $\begin{array}{l}\text { Short form of the } \\
\text { Marlowe-Crowne } \\
\text { social desirability } \\
\text { scale (M-C) prior } \\
\text { to receiving the } \\
\text { intervention }\end{array}$ & Edmonds et al. (7) & $\begin{array}{l}\text { Edmonds et al. (2011) revisaron } 13 \text { artículos con el fin de evaluar el sesgo de } \\
\text { respuesta, sugiere que en las enfermeras el atractivo social tiene puntajes superiores } \\
\text { y están relacionados con un menor auto-informes de agotamiento. La escala abreviada } \\
\text { demuestra una aceptable nivel de fiabilidad }(\mathrm{r}=0,76) \text { y una buena validez concurrente } \\
\text { con la forma completa de la prueba }(\mathrm{r}=0,93) \text {. }\end{array}$ \\
\hline $\begin{array}{lr}\text { Draw-a-Pers on - } & \\
\text { in-the-Rain } & \text { Art } \\
\text { Assessment } & \text { (PIR). } \\
\text { Desarrollado por } \\
\text { Carney en } 1992\end{array}$ & Kravits et al. (8) & $\begin{array}{l}\text { Se utiliza para aumentar la información obtenida de la MBI. El PIR revela la interacción } \\
\text { entre la intensidad de los factores de estrés y la disposición de los recursos de } \\
\text { afrontamiento y su potencial para ser eficaz. }\end{array}$ \\
\hline The SF-12v2 & Goodman y Schorling (31) & Es un cuestionario de 12 ítems para medir la percepción de salud física y mental. \\
\hline $\begin{array}{l}\text { Medida desarrollada } \\
\text { por el } \\
\text { Ewers }\end{array}$ & \multirow{2}{*}{ Ewers et al. (33) } & $\begin{array}{l}\text { El objetivo de la escala fue identificar si los sujetos tenían actitudes realistas y positivas } \\
\text { hacia el cuidado de sus pacientes, lo que llevaría a respuestas a las dificultades que } \\
\text { resultan de la enfermedad. }\end{array}$ \\
\hline $\begin{array}{l}\text { Prueba de } \\
\text { conocimientos }\end{array}$ & & $\begin{array}{l}\text { Objetivo medir los aprendizajes y conocimientos sobre la esquizofrenia y enfoques en } \\
\text { psicología. }\end{array}$ \\
\hline
\end{tabular}




\begin{tabular}{|c|c|c|}
\hline $\begin{array}{l}\text { Profesional } \\
\text { Inventario } \\
\text { Autoimagen } \\
\text { Professional Self } \\
\text { Concept Inventory } \\
\text { (PSCI) }\end{array}$ & & $\begin{array}{l}\text { Fue desarrollado para evaluar el desarrollo del autoconcepto profesional en enfermería. } \\
\text { Es un inventario de evaluación de tip o Likert de } 4 \text { puntos que consiste en } 36 \text { elementos } \\
\text { con tres subescalas. Estas subescalas se llaman "Satisfacción profesional "," competencia } \\
\text { profesional "y "Actitud profesional y habilidad " }\end{array}$ \\
\hline $\begin{array}{l}\text { Minnesota } \\
\text { Inventario de } \\
\text { satisfacción en el } \\
\text { trabajo } \\
\text { Minnesotta } \\
\text { Satisfaction } \\
\text { Questionnaire } \\
\text { (MSQ) }\end{array}$ & Sabanciogullari y Dogan (32) & $\begin{array}{l}\text { Se les pide a los individuos que calificaran su nivel de satisfacción con cada una de los } \\
20 \text { necesidades relacionadas con el trabajo, en una escala de } 5 \text { puntos que van desde } \\
\text { "no satisfactorio: } 1 \text { hasta" extremadamente satisfactorio: 5. Las respuestas a los } 20 \\
\text { elementos pueden ser sumadas para producir la puntuación de satisfacción general. } \\
\text { Adicionalmente, los componentes intrínsecos y extrínsecos de la satisfacción pueden } \\
\text { ser examinados. A medida que aumenta la puntuación también lo hace la satisfacción } \\
\text { en el trabajo. }\end{array}$ \\
\hline $\begin{array}{l}\text { Escala de Apoyo } \\
\text { social }\end{array}$ & \multirow{4}{*}{ Le Blanc et al. (34) } & $\begin{array}{l}\text { El estudio utilizó dos escalas: Peeters y Peeters, Buunk y Schaufeli de } 1993 \text { y } 1994 \text {, } \\
\text { se ocupan de la percepción de apoyo social recibido por los compañeros de trabajo y } \\
\text { del supervisor directo: apoyo emocional, apoyo evaluativo, apoyo informativo y apoyo } \\
\text { instrumental. Se evalúa cada ítem con una escala de } 1 \text { (nunca) a } 5 \text { (siempre). El alpha } \\
\text { de Cronbach fue de } 0,64 \text {. }\end{array}$ \\
\hline $\begin{array}{l}\text { Escala para } \\
\text { medir el nivel } \\
\text { de participación } \\
\text { percibida en la toma } \\
\text { de decisiones }\end{array}$ & & $\begin{array}{l}\text { Escala construida por Van Veldhoven, Meijman, Broersen y Fortuin en } 1997 \text {, pero } \\
\text { modificada por los autores para ajustarse a los objetivos y muestra del estudio. Se } \\
\text { califican en una escala de 1(nunca) a } 4 \text { (siempre). El alpha de Cronbach para esta escala } \\
\text { fue de } 0,86\end{array}$ \\
\hline $\begin{array}{l}\text { Escala para el } \\
\text { control del trabajo }\end{array}$ & & $\begin{array}{l}\text { Escala de cuatro ítems, propuesta por Biessen y De Gilder en 1993. Evalúa en que forma } \\
\text { los individuos tienen la libertad de decidir la organización de su trabajo y la forma de } \\
\text { trabajar. La escala de los ítems va de 1(nada) a } 5 \text { (completamente). El alpha de Cronbach } \\
\text { fue de } 0,81 \text {. }\end{array}$ \\
\hline $\begin{array}{l}\text { Escala de demandas } \\
\text { laborales }\end{array}$ & & $\begin{array}{l}\text { El estudio utilizó otras escalas, basada en instrumentos originales de Furda en 1995, } \\
\text { Herschbach en 1992, Kleiber en 1992, y aplicadas por Pascale y Le Blanc: } \\
\text { - Escala que evalúa la frecuencia en que el individuo se enfrenta a exigencias como un } \\
\text { alto ritmo de trabajo, grandes cantidades de trabajo, y jornadas extensas. } \\
\text { - Problemas en la llamada interacción con los pacientes } \\
\text { - Confrontación con la muerte } \\
\text { - Identificación con los pacientes } \\
\text { Las escalas se combinaron en una sola, exigencias emocionales en el trabajo con un } \\
\text { alpha de Cronbach de } 0,78 \text {. }\end{array}$ \\
\hline $\begin{array}{l}\text { The Centre for } \\
\text { Epidemiologic } \\
\text { Studies Depression } \\
\text { Scale (CES-D) }\end{array}$ & Quenot et al. (35) & Evalúa síntomas de depresión a través de 20 preguntas. \\
\hline
\end{tabular}

Fuente: elaboración propia 
ISSN 1794-9831

E-ISSN 2322-7028

Vol. 14 No. 1

Ene - Jun 2017

Cúcuta, Colombia
Los estudios utilizaron técnicas cualitativas como planes de bienestar personalizado, que documentaron las intenciones y las estrategias que cada participante manejó para ayudar a guiar nuevas prácticas de autocuidado positivo (8).

Estas herramientas se fundamentaron en el análisis de contenido (8) de registros de variables dicotómicas como: registros de acontecimientos de la vida durante un periodo determinado -divorcio, enfermedad, muerte, incapacidad por enfermedad, conflicto con el trabajo, tratamiento o psicoterapia con psicólogo o psiquiatra, número de horas de trabajo y reducción de las horas de trabajo (30)-.

También se utilizó la entrevista semiestructurada con análisis temático (37) y el cuestionario semi-estructurado para evaluar las percepciones individuales de los cambios de salud y calidad de vida (36).

\section{Intervenciones}

En relación con las intervenciones, en la Tabla 2 se sintetizan las reportadas por los artículos, las cuales se centran en propiciar cambios en el individuo. Tres artículos $(6,14,38)$ reportan estrategias organizacionales. Al respecto, la preparación para el estrés es un predictor que atenúa los efectos de $\mathrm{SB}$, requiere de aproximaciones individuales para desarrollar efectivamente el afrontamiento y la relajación que ayudan a aliviar el EE, pero no se reporta utilidad para los otros dos componentes de DP y PA.

Ortega y López (6) reportaron el impacto de una estrategia grupal para identificar los problemas y estrategias en relación al SB, en la reducción de la $\mathrm{EE}$, incluso tras un seguimiento de 6 a 12 meses. Sin embargo, el autor sugiere la realización de estudios longitudinales para comprobar el impacto de las estrategias, así como investigaciones con grupos de control.

Ourcilleón et al. (14) mencionan que realzar la empatía y el empoderamiento, además del entrenamiento en habilidades comunicativas, mejora la PA, ya que aumenta la autoconfianza del personal. Para desarrollar estrategias de prevención se debe partir de la capacidad de autorregulación de los individuos, que les permite determinar la forma de enfrentar las diferentes situaciones que se presentan. Se debe incluir la opinión de los trabajadores en cualquier tipo de intervención, en pro de una gestión de calidad, para aumentar la competencia profesionales con el menor costo emocional.

La revisión de Edwards y Burnard (16) evidenció efectividad en la reducción de los niveles de estrés. No obstante, no es posible determinar específicamente cuáles son las intervenciones o técnicas más efectivas. Las acciones que desarrollan las enfermeras para manejar el estrés varían de acuerdo con su experiencia laboral. Las enfermeras con mínima experiencia (3 y 6 meses) realizan actividades con el fin de alejar su atención del estrés, mientras que las enfermeras con mayor experiencia utilizan el aislamiento y competencia laboral como mecanismo de defensa (38).

Nueve artículos $(7,9,14,15,29,30,34,35,38)$ señalan que las intervenciones se realizaron también de forma grupal. El trabajo en grupo es una herramienta útil para promover aproximaciones entre los participantes $\mathrm{y}$, en relación con sus situaciones comunes, facilita la realización de la tarea profesional al contribuir con la satisfacción en el ambiente de trabajo (39).

Cuatro artículos $(13,15,35,37)$ reportan, como estrategias de afrontamiento, la discusión de factores de riesgo para el SB, técnicas para el manejo del estrés, resolución de problemas, relajación y apoyo social, siendo esta última la más utilizada.

Popp (28) señala que las estrategias de afrontamiento o coping hacen referencia a las respuestas de las personas en determinadas situaciones, cuando no disponen de los recursos para ello. Según el estudio de Kravits et al. (8), el afrontamiento es un proceso para promover supervivencia frente a una amenaza. Las estrategias de afrontamiento son patrones de comportamiento influenciados por los rasgos de personalidad, patrones históricos de relaciones y los factores de estrés situacional.

Estas estrategias pueden promoverse mediante la estimulación de la creencia en el poder personal para controlar las circunstancias de la vida, la creación de objetivos alcanzables y generar un estado de ánimo positivo (8). Según Popp (28), se distinguen 
tres dimensiones: las centradas en el problema, en la emoción o en la evaluación. Montoya y Moreno (40) refieren tres tipos de afrontamiento: cognitivo, conductual y afectivo.

Para Berrios et al. (41), las estrategias de afrontamiento centradas en el problema y en el control de sus propias emociones, así como la detección e interpretación adecuada de las emociones de los demás, disminuyen la vulnerabilidad para desarrollar SB. La reevaluación positiva consiste en representar el problema, enfatizando sólo en los aspectos positivos que contrarresten lo negativo y permitan un afrontamiento optimista (40). Es una estrategia de afrontamiento de estilo activo y se asocia con menor despersonalización y menor probabilidad de desarrollar el SB (13).

Según Popp (28), el afrontamiento focalizado en el problema conduce a un mejor ajuste y adaptación ante los estresores de la vida y a experimentar menos síntomas psicológicos. Davis et al. (42) aluden que las estrategias de afrontamiento eficaces tienen un impacto positivo al reducir el desgaste entre las enfermeras.

Por el contrario, las estrategias de afrontamiento de evaluación como la evitación y la emoción, Berrios et al. (41), determinan un mayor riesgo de desarrollar el SB, en concordancia con baja autoeficacia y con ausencia de control emocional. Para Montoya y Moreno (40), la evitación emocional es la capacidad de controlar y bloquear las emociones negativas que surgen como consecuencia de la situación estresante y la negación es la tendencia a suprimir la situación problemática, como una manera de evitar los efectos negativos consecuentes con la misma. Estas hacen parte, según Contreras et al. (13), de las estrategias de afrontamiento de estilo paliativo que favorecen la aparición del $\mathrm{SB}$, al relacionarse con mayor carga emocional, falta de realización personal y despersonalización.

Siete artículos $(7,914,15,29,35,38)$ indicaron otras estrategias como el trabajo en grupo y la estrategia de afrontamiento de apoyo social, ésta última referida a las transacciones entre las personas que conforman una red de apoyo al individuo, las que se brindan ayuda entre sí y satisfacen sus necesidades básicas.
Beltrán et al. (43) mencionan que existen varias conductas relacionadas tales como demostrar afecto e interés, escuchar, prestar objetos materiales, ofrecer consejos, ofrecer ayuda y manifestar empatía. La teoría que concibe el apoyo social con efectos positivos potenciales frente al SB se origina en la psicología y en la sociología: en el ambiente laboral las relaciones interpersonales basadas en una buena comunicación se perciben como apoyo social y amortiguan los efectos negativos del estrés sobre la salud (43).

Dos artículos $(16,35)$ presentaron como estrategia el entrenamiento en habilidades comunicativas, como sesiones de grupo de afrontamiento $\mathrm{y}$ la psicoeducación. Damiani (44) indica que el entrenamiento en habilidades comunicativas se refiere a aspectos que mezclan rasgos de carácter, actitudes y cualidades emocionales, además de habilidades generales como expresión, relación empática y observación conductual

También menciona que, en esta última, se incluyen la escucha atenta, la percepción de estados de ánimo y sentimientos en los que están presentes signos no verbales como la disposición, las actitudes formales o de rechazo, los estados emocionales o índices de cansancio, el aburrimiento o, por el contrario, el interés, etc. (44).

Vega y González (45) mencionan que las sesiones de grupo de apoyo son definidas como los lazos que permiten la integración psicológica y física de una persona, muestran a los individuos como parte de una red de relaciones sociales y estas se vuelven importantes cuando proveen el apoyo necesario en situaciones de crisis en la vida en general. Davis et al. (42) sugieren que el fuerte apoyo social está vinculado con niveles inferiores de agotamiento emocional y DP.
ISSN 1794-9831

E-ISSN 2322-7028

Vol. 14 No. 1

Ene - Jun 2017

Cúcuta, Colombia

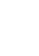


Estrategias que modulan el síndrome de Burnout en enfermeros (as): una revisión bibliográfica • Luz Dalila Vargas-Cruz,Carmen Lucía Niño-Cardozo,Jessica Yurlay Acosta-Maldonado

ISSN 1794-9831

E-ISSN 2322-7028

Vol. 14 No. 1

Ene - Jun 2017

Cúcuta, Colombia
Tabla 2. Artículos sobre estrategias en enfermeras para disminuir o prevenir el SB.

\begin{tabular}{|c|c|c|}
\hline Estudio & Tipo de Estudio & Estrategias de prevención, control y reducción \\
\hline $\begin{array}{l}\text { Ortega y López } \\
\text { (6) }\end{array}$ & $\begin{array}{l}\text { Revisión de literatura: } \\
\text { personal sanitario }\end{array}$ & $\begin{array}{l}\text { Cuatro objetivos: "reducir o eliminar las demandas laborales; cambiar las metas, preferencias y } \\
\text { expectativas personales; incrementar los recursos de las personas ante las demandas y proveer } \\
\text { estrategias acordes a las características del SB" (6). } \\
\text { Técnicas individuales de tipo físico, emocional y conductual: relajación, biofeedback, técnicas } \\
\text { cognitivas como reestructuración cognitiva, resolución de problemas, entrenamiento de la } \\
\text { asertividad, fomentar habilidades de afrontamiento y técnicas de autocontrol (6). } \\
\text { Estrategias a nivel organizacional: incrementar la autonomía del trabajo, planificar un horario } \\
\text { flexible por parte del trabajador, fomentar la participación del trabajador en la toma de decisiones, } \\
\text { mejorar los niveles de calidad del ambiente físico, enriquecer la calidad del trabajo, asignación } \\
\text { de tareas al grupo, limitar el número de horas y de pacientes y plantearse objetivos claros en el } \\
\text { equipo de trabajo (6). }\end{array}$ \\
\hline $\begin{array}{l}\text { Ourcilleón et } \\
\text { al. (14) }\end{array}$ & $\begin{array}{l}\text { Revisión sistemática de la } \\
\text { literatura, profesionales de } \\
\text { enfermería en UCI de } 1995 \\
\text { a } 2005\end{array}$ & $\begin{array}{l}\text { Individuales: entrenamiento en la solución de problemas, conductas que eliminen las fuentes de } \\
\text { estrés, entrenamiento en asertividad y manejo del tiempo (14). } \\
\text { Grupal: apoyo social en el trabajo por compañeros y supervisores, refuerzo social y } \\
\text { retroalimentación, trabajo en equipo, funciones y roles de enfermería (14). } \\
\text { Organizacional: programas para mejorar el ambiente físico y el clima de la organización, mejorar } \\
\text { la comunicación, disminuir la burocracia (14). }\end{array}$ \\
\hline $\begin{array}{l}\text { Edwards } y \\
\text { Burnard (16) }\end{array}$ & $\begin{array}{l}\text { Revisión de literatura de } \\
\text { estrés en enfermeras en } \\
\text { salud mental. } 18 \text { estudios } \\
\text { incluyeron el SB. }\end{array}$ & $\begin{array}{l}\text { Talleres de manejo del estrés } \\
\text { Asistencia a un curso de } 15 \text { semanas, en habilidades terapéuticas, basada en el modelo de tres } \\
\text { etapas de Egan. } \\
\text { Intervención psicosociales } \\
\text { Acomodación creativa } \\
\text { Supervisión clínica a las enfermeras } \\
\text { Las estrategias de afrontamiento más frecuentes fueron de apoyo social y los factores asociados } \\
\text { con un mayor uso de estas fueron: el ser mujer, mayor experiencia, se mayor y ambiente de trabajo. }\end{array}$ \\
\hline $\begin{array}{l}\text { Ewers et al. } \\
(33)\end{array}$ & $\begin{array}{ll}\text { Cuantitativa, } & \text { cuasi- } \\
\text { experimental: } & \text { grupo } \\
\text { control } & \end{array}$ & $\begin{array}{l}\text { Intervención Psicosocial PSI ( } 6 \text { meses) conocimientos y actitudes y niveles de SB } 20 \text { días de } \\
\text { entrenamiento. }\end{array}$ \\
\hline $\begin{array}{l}\text { Sabanciogullari } \\
\text { y Dogan (32) }\end{array}$ & $\begin{array}{l}\text { Cuantitativa, } \\
\text { experimental }\end{array}$ & $\begin{array}{l}\text { Programa de desarrollo de la identidad profesional } 10 \text { sesiones una vez por semana. Se centra en } \\
\text { el pensamiento positivo, la fijación de objetivos profesionales y un plan de desarrollo de la propia } \\
\text { imagen profesional. }\end{array}$ \\
\hline $\begin{array}{l}\text { Contreras et al. } \\
\text { (13) }\end{array}$ & Descriptivo, correlacional & $\begin{array}{l}\text { Estrategias de afrontamiento: Focalización en la solución de problemas y reevaluación positiva } \\
\text { y religión }\end{array}$ \\
\hline $\begin{array}{l}\text { Vásquez et al. } \\
\text { (15) }\end{array}$ & $\begin{array}{l}\text { Cuasi-experimental grupo } \\
\text { experimental y control }\end{array}$ & $\begin{array}{l}\text { Intervención psicoterapéutica grupal, de forma intensiva durante } 3 \text { días en alojamiento rural, } 50 \\
\text { horas de convivencia en grupo (15). } \\
\text { Aprendizaje de técnicas para manejo del estrés; estilos de afrontamiento positivos y negativo, } \\
\text { counselling y comunicación asertiva, técnicas: } \\
\text { - Entrenamiento en focalización sensorial y respiración profunda } \\
\text { - Counselling: escucha activa, respuesta empática, respuestas de validación, preguntas } \\
\quad \text { exploratorias, reformulación, persuasión, confrontación, mensajes validantes. } \\
\text { - Técnicas para resolución de conflictos, pedir disculpas, cambios de comportamientos (15). }\end{array}$ \\
\hline Italia et al. (9) & $\begin{array}{l}\text { Cuasi experimental, grupo } \\
\text { experimental y grupo de } \\
\text { control }\end{array}$ & $\begin{array}{l}\text { Programa de apoyo en grupo, objetivo aumentar la colaboración y las relaciones a través de la } \\
\text { creatividad y el arte. } 4 \text { meses, } 13 \text { reuniones semanales, a cargo de psicólogo y terapeuta en arte y } \\
\text { psicología. Técnicas: } \\
\text { - Psicodrama (comunicación) } \\
\text { - Terapia de juego (confort) } \\
\text { - Técnicas de relajación Ericksoniana (ansiedad) }\end{array}$ \\
\hline $\begin{array}{ll}\text { Günusën } & \text { y } \\
\text { Üstün }(29)\end{array}$ & $\begin{array}{l}\text { Ensayo Controlado } \\
\text { Aleatorio (ECA), }\end{array}$ & $\begin{array}{l}\text { Programa de } 7 \text { sesiones, dentro del hospital pero en un ambiente diferente. } \\
\text { Grupo de afrontamiento: estrategias de afrontamiento cognitivas y método de resolución de } \\
\text { problemas. } \\
\text { Grupo de apoyo social: proporcional apoyo, información, sentido de pertenencia y ambiente para } \\
\text { compartir experiencias. Se utilizó el modelo de reflexión. }\end{array}$ \\
\hline
\end{tabular}




\begin{tabular}{|c|c|c|}
\hline $\begin{array}{l}\text { Günusën et al. } \\
\text { (37) }\end{array}$ & $\begin{array}{l}\text { Investigación cualitativa } \\
\text { descriptiva, análisis de } \\
\text { contenido cualitativo }\end{array}$ & Indagación de los motivos por los que no asistieron o terminaron el Programa \\
\hline $\begin{array}{l}\text { Edmons et al. } \\
\text { (7) }\end{array}$ & No refiere & $\begin{array}{l}\text { El Programa de Cuidado al Cuidador Profesional (CPCP) aborda los problemas los profesionales } \\
\text { que trabajan con los enfermos terminales. } 1 \text { día de sesión Empezó en } 2003 . \\
\text { - Modelo de adaptación } \\
\text { - Consecuencias de SB y estrategias para manejar el estrés } \\
\text { La intervencios de reducción de estrés: relajación, imaginación a los } 6 \text { meses } \\
\text { Intervención en grupo. }\end{array}$ \\
\hline $\begin{array}{l}\text { Kravits et al. } \\
(8)\end{array}$ & No refiere & $\begin{array}{l}\text { Programa psico-educativo ( } 6 \text { horas) de estrategias de autocuidado para desarrollar en enfermeras } \\
\text { planes de manejo del estrés, basadas en estrategias de afrontamiento adaptativo, intervenciones: } \\
\text { - Discusión de factores de riesgo } \\
\text { - Técnicas de relajación } \\
\text { - } \quad \text { Exploración a través del arte. }\end{array}$ \\
\hline $\begin{array}{l}\text { Goodman } y \\
\text { Schorling (31) }\end{array}$ & No refiere & $\begin{array}{l}\text { Programa de Atención plena para proveedores de servicios de salud, por } 8 \text { semanas, } 2,5 \text { horas a la } \\
\text { semana, se basa en reducción de estrés. Mindfulness Based Stress Reduction (MBSR). Atención } \\
\text { plena (movimiento consciente, comunicación: } \\
\text { - } \quad \text { Exploración del cuerpo } \\
\text { - Movimiento consciente } \\
\text { - Meditación caminando } \\
\text { - Meditación sentada }\end{array}$ \\
\hline $\begin{array}{l}\text { Isaksson et al. } \\
(30)\end{array}$ & No refiere & $\begin{array}{l}\text { Curso en el centro de recursos para el personal de salud. ( } 5 \text { días) Basado en la teoría cognitiva: } \\
\text { Técnicas: } \\
\text { - } \quad \text { Ejercicios de relajación } \\
\text { - } \quad \text { Actividad física } \\
\text { - } \quad \text { Trabajo en grupo } \\
\text { - } \quad \text { Asesorinación guiada } \\
\quad \text { individual }\end{array}$ \\
\hline $\begin{array}{l}\text { Rodrigues et al. } \\
\text { (36) }\end{array}$ & $\begin{array}{l}\text { Estudio pre-post cuasi- } \\
\text { experimental }\end{array}$ & Programa AFT: actividad física, 10 minutos, cinco días por semana, durante tres meses \\
\hline $\begin{array}{l}\text { Le Blanc et al. } \\
\text { (34) }\end{array}$ & Estudio cuasi-experimental & Programa Cuídate: 6 sesiones mensuales de 3 horas. Apoyo social, control y participación \\
\hline $\begin{array}{l}\text { Quenot et al. } \\
\text { (35) }\end{array}$ & Estudio monocéntrico & $\begin{array}{l}\text { Estrategia intensiva de comunicación a través de grupos de trabajo y participación en conferencias: } \\
\text { - Mejorar la comunicación entre equipo de trabajo. } \\
\text { - } \quad \text { Individuo y estrategias personales de afrontamiento. Ajustes psicológicos individuales } \\
\text { - Enfoque colectivo y multidisciplinar. }\end{array}$ \\
\hline
\end{tabular}

\section{Fuente: elaboración propia}


ISSN 1794-9831

E-ISSN 2322-7028

Vol. 14 No. 1

Ene - Jun 2017

Cúcuta, Colombia
Albarrán y Macías (46) mencionan como estrategia de autocuidado la psico-educación, que es un modelo que hace referencia a la educación e información que se da a una persona, especialmente con un trastorno psiquiátrico, sobre todo aquello que afecta la salud mental -consecuencias, intervenciones, actividades a realizar- para minimizar su incidencia.

Al respecto, Vásquez et al. (15) relacionan la psico-educación como herramienta de un programa multicomponente de trabajo psicoterapéutico, desde el esquema de la intervención para la Inoculación del Estrés, utilizando técnicas cognitivo-conductuales y a través de un trabajo en grupo de apoyo social. Ewers et al. (33), a pesar de las limitaciones metodológicas del estudio, sugieren que un programa de psicoeducación permite a las enfermeras conocer mejor a sus pacientes y administrarles formas de intervención más efectivas, con lo cual se experimenta menos sentimiento de frustración y desesperanza, facilitando la prevención o atenuación del agotamiento.

Sobre la estrategia de la identidad profesional, Sabanciogullari y Dogan (32) señalan que el desarrollo de esta puede ayudar a enfermería a alcanzar el estatus profesional, aumentado su compromiso, la mejora de sus prácticas, la mejora en la satisfacción laboral y la reducción de los niveles de Burnout.

Al respecto, es pertinente mencionar el autocuidado definido por Orem como una acción que "refuerza la participación activa de las personas en el cuidado de su salud, como responsables de decisiones que condicionan su situación, coincidiendo de lleno con la finalidad de la promoción de la salud" (47). León (47) sugiere que los profesionales de enfermería, como defensores de la salud, deben fomentar en su propia persona el autocuidado, de tal manera que propicien el bienestar holístico para desarrollar una personalidad terapéutica que transmita e inspire salud.

Otras estrategias de afrontamiento que utilizan las enfermeras para la modulación del SB son la religión y el entrenamiento en la solución de problemas a través de la reevaluación positiva (13). A propósito, Montoya y Moreno (40) señalan que la religión hace referencia a la persona que se vale de la oración y de sus creencias religiosas para solucionar el problema; hace parte de los doce tipos de estrategias de afrontamiento.
Montoya y Moreno (40) también indican que el entrenamiento en la solución de problemas implica una flexibilidad cognitiva para buscar soluciones orientadas a la resolución del problema en el momento que se presente y la reevaluación positiva consiste en resignificar el problema, enfatizando sólo en los aspectos positivos que contrarresten los negativos y permitan un afrontamiento optimista. De acuerdo con Chang y Chan (48), el optimismo contribuye a un mayor bienestar psicológico y está asociado a la reducción del SB.

Rodrigues et al. (36) centraron sus estrategias en la actividad física en el trabajo, elemento condicionante de la calidad de vida, de la salud y del bienestar, que produce efectos beneficiosos tanto físicos como psicológicos; sin embargo, este estudio no mostró cambios significativos en el agotamiento o estrés laboral.

Como técnicas utilizadas para reducir el SB los artículos reportaron: role playing, psicodrama, asertividad, espiritualidad, ejercicios prácticos para interiorizar, counselling, autorreflexiones, análisis de casos, cooperación grupal, prácticas de focalización sensorial, respiración, relajación muscular, ejercicios físicos, paseos al aire libre, meditación e inteligencia emocional, entre otras.

El psicodrama hace parte de las psicoterapias grupales. Al respecto, Espina (49) sostiene que "el yo proviene de los roles que el sujeto desempeña a lo largo de su vida y no al revés, como afirma el psicoanálisis. La teoría de los roles se instaura como una serie de círculos concéntricos; en el centro se encuentran los roles psicosomáticos (dormilón, comilón, llorón). Por fuera, los roles familiares (padres, hermanos, vecinos, compañeros)". Esta técnica se centra más en el encuentro interhumano que en las interioridades psíquicas del ser humano, donde interesa la cantidad y calidad de los mensajes, su finalidad dentro del grupo, la posición de los participantes, la consideración del grupo como una totalidad y las transferencias laterales entre los miembros del grupo (49).

De acuerdo con Terroni (50), el entrenamiento en asertividad significa "afirmar con certeza alguna cosa y, por lo tanto, se considera que la persona asertiva es aquella que puede enunciar con certeza sus opiniones y deseos, juega un papel importante 
en las interacciones grupales, ya que constituye la habilidad o destreza a la hora de emitir opiniones y en los procesos de influencia grupal".

La espiritualidad, de acuerdo con Davis et al. (42), engloba el concepto de auto-trascendencia o hallazgo de sentido a la vida y a la muerte. Las enfermeras (os) que implementan la auto- trascendencia para poder dirigir los sentimientos de desesperación e impotencia, son menos propensos a experimentar agotamiento. En el estudio de Günusën y Üstün (29) se incrementó la EE después de 6 meses de realizada la intervención y los autores sugieren que este incremento puede estar relacionado con no haber involucrado la dimensión espiritual en el programa que se implementó para el control del Burnout.

La inteligencia emocional (IE), según Berrios et al. (41), "se define como el resultado de una interacción adaptativa entre las emociones y la propia cognición, en la que se incluye la habilidad para percibir, asimilar, entender y manejar las emociones propias y las de los demás. Este concepto adquiere importancia en las profesiones asistenciales como enfermería, dada la habilidad que debe poseer el profesional para controlar sus propias emociones y detectar, interpretar y manejar correctamente las emociones de los demás" (41).

Berrios et al. (41) mencionan que "las enfermeras (os) que adoptaban un estilo de liderazgo basado en competencias propias de la inteligencia emocional informaban de menor cansancio emocional y menos síntomas psicosomáticos, de mayor salud emocional, más satisfacción con su trabajo y mayor conocimiento de las necesidades de sus pacientes que los profesionales que no basaban su estilo de liderazgo en dichas competencias". Görgens y Brand (51) refieren que una inteligencia emocional superior está significativamente relacionada con un menor estrés y SB.

\section{Resultados de las estrategias en la reducción del SB}

En lo concerniente a los resultados de las intervenciones en la reducción del $\mathrm{SB}$, seis artículos reportaron disminución o ausencia del SB después de las intervenciones para las tres dimensiones EE, DP y PA $(8,9,30,31,33,35)$, cinco descenso en $\operatorname{EE~}(7$,
$15,29,32,34)$, dos disminución de $\operatorname{EE}$ y $\operatorname{DP}(7,34)$ y uno aumento en la PA y disminución de la EE (32). Un artículo (36) indicó no haber cambios en las tres dimensiones después de la intervención.

Un artículo (7) informó sobre el efecto positivo en EE y DP al mes de la intervención; otro, disminución del SB al año de la intervención (30); otro reveló el efecto positivo en DP y PA al año post intervención, pero se debilitaron la ansiedad y la EE (15). Dos artículos indicaron aumento en la EE. Vásquez et al. (15) señalaron que, después de un tiempo, puede aumentar sin sobrepasar la puntuación inicial. En Günusën y Üstün (29) aumentó a los 6 meses. Al parecer, estas intervenciones no pueden mantener su eficacia a largo plazo, por lo tanto deben ser repetidas periódicamente.

Respecto a la DP, los estudios $(15,29)$ no mostraron cambios significativos con la implementación de la estrategia en entrenamiento en la solución de problemas, mientras que la psico-educación sí mostró una reducción de la DP de 13\% al 9\% pos intervención; y PA no mostró cambios significativos pos intervención.

El EE es la base para el SB o primera etapa de agotamiento (29) y refleja el grado de fatiga experimentada por el participante (30). Las altas puntuaciones en EE están asociadas con un mayor riesgo de agotamiento y conducen a mayor sensación de despersonalización y disminución de los sentimientos de realización personal $(8,29)$.

Italia et al. (9), Ourcilleón et al. (14), Görgens y Brand (51), Goodman y Schorling (31), Edmons et al. (7), Kravits et al. (8), Quenot et al. (35), Vázquez et al. (15), Contreras et al. (13) y Rodrigues et al. (36) no discriminan las estrategias por variable, éstas son usadas en contexto para el SB. Las estrategias que se mencionan con mayor frecuencia en los artículos son el afrontamiento por apoyo social de compañeros de trabajo y supervisores. De otro lado, la psicoeducación representó grandes cambios en EE pos intervención, la reducción más significativa fue de un $26 \%(8)$.

En cuanto a la DP hay controversia en la efectividad de la intervención en esta categoría; en el estudio realizado por Günusën y Üstün (29) no se presentaron
ISSN 1794-9831

E-ISSN 2322-7028

Vol. 14 No. 1

Ene - Jun 2017

Cúcuta, Colombia 
ISSN 1794-9831

E-ISSN 2322-7028

Vol. 14 No. 1

Ene - Jun 2017

Cúcuta, Colombia cambios significativos con las intervenciones propuestas. "El apoyo social representó una reducción inmediata de la EE, en cuanto a la DP y PA no se mostraron cambios significativos pos-intervención, éste facilitará que el sujeto evalúe de forma adecuada su entorno social, así como sus actitudes hacia los demás, esto nos explica cómo el apoyo social puede desencadenar o facilitar los efectos del SB en el trabajo" (29).

Las actividades realizadas ayudaron a los profesionales a reducir la EE significativamente, en las variables de DP y PA no se reportaron cambios significativos (29).

En el estudio de Contreras (13) se observó incremento en el uso de estrategias de afrontamiento con reevaluación positiva y religión como estrategias predominantesenmujeres, demenoredady enfermeras; sin embargo, las estrategias de focalización en la solución de problemas y de reevaluación positiva son más influyentes en el incremento de la calidad de vida $\mathrm{y}$, con esto, en la reducción o protección frente a los síntomas de Burnout.

En coherencia con la estrategia de afrontamiento de la religión, la espiritualidad puede ser usada por el individuo para desarrollar su autocuidado en la prevención del SB de una manera consciente; durante su desarrollo de debe confiar y creer en que existe algo superior a uno mismo (52). Esto coincide con el trabajo de Da Silva et al. (53) en el que destaca que los cuidadores buscaban la espiritualidad como medio de enfrentamiento, contribuyendo a superar sentimientos negativos y a encontrar sentido a la tarea.

En cuanto a la evitación emocional, estrategia de afrontamiento observada por Contreras (13), no reporta influencia en la modulación del Burnout. Popp (28) la considera como menos eficiente y efectiva por tener un "menor control sobre la emoción y es usada en situaciones que el sujeto valora como amenazantes, lesivas, desafiantes y sin posibilidad de modificación; esta puede dañar la salud al impedir conductas adaptativas relacionadas con la saludenfermedad".

Dos artículos $(15,35)$, presentaron como estrategias el entrenamiento en habilidades comunicativas, las cuales mostraron para Quenot et al. (35) una reducción del SB del 50\% y aumento significativo de la sensación de la PA. El estudio de Vázquez et al. (15) mostró mejoría significativa de la ansiedad y de la PA, efecto positivo sobre EE; al año de las intervenciones siguió el efecto positivo en la PA y en la DP, en ansiedad y EE se debilitaron.

Para Vázquez et al. (15), no existe una estrategia terapéutica simple que, por sí sola, resulte efectiva para tratar el desgaste profesional en personal de oncología; de ahí la importancia de las intervenciones de tipo multicomponente. Laurenţiu et al. (27) consideran que los efectos de las estrategias, en general, son modestos y duraderos y se necesitan nuevas estrategias más adaptadas para reducir el desgaste y mejorar el efecto de las intervenciones. Por otro lado, Taormina y Law (54) apuntan a que el enfoque estándar para la lucha contra el desgaste entre las enfermeras se dirige a la mejora en gestionar el propio estrés

\section{CONCLUSIONES}

Los artículos consultados reportan como instrumento base para la medición el MBI e involucran otros que complementan el análisis del desgaste con ansiedad, depresión, estrés, estrategias de afrontamiento, calidad de vida, salud mental, conocimientos, autoconcepto profesional, demandas laborales y apoyo social en el trabajo. Las mediciones realizadas permiten evaluar el impacto de las intervenciones realizadas con relación al Burnout.

La mayoría de los artículos reporta estrategias para el SB, sin distinción de las variables propuestas por Maslach y Jackson de cansancio emocional, despersonalización y falta de realización personal; sin embargo, se evidencian diferencias en los resultados frente a cambios positivos en cansancio emocional a diferencia de las otras variables. Las estrategias reportadas con mayor frecuencia en los artículos fueron la psico-educación y el apoyo social por compañeros de trabajo y supervisores.

Las intervenciones a realizar deben ser de tipo multicomponente, que involucren los aspectos espiritual, profesional, inteligencia emocional y liderazgo. Las estrategias que implementaron el apoyo social y la supervisión reportaron resultados positivos en la modulación del Burnout. 
Como profesionales de enfermería es preciso implementar el autocuidado para prevenir el Burnout; las técnicas utilizadas hacen parte del conocimiento disciplinar y la teoría de enfermería proporciona los elementos necesarios para realizar acciones de cuidado entre colegas.

\section{CONFLICTO DE INTERESES}

Las autoras declaran no tener ningún conflicto de intereses.
E-ISSN 2322-7028

Vol. 14 No. 1

Ene - Jun 2017

Cúcuta, Colombia 
ISSN 1794-9831

E-ISSN 2322-7028 Vol. 14 No. 1

Ene - Jun 2017 Cúcuta, Colombia

\section{REFERENCIAS BIBLIOGRÁFICAS}

1. Ramírez M, Lee S. Síndrome de Burnout entre hombres y mujeres medido por el clima y la satisfacción laboral. Polis [Internet]. 2011 [consultado 15 de febrero de 2015]; 10(30); 431-446. Disponible en: http://www.redalyc. org/articulo.oa? id $=30521366020$

2. Maslach C, Jackson S. The measurement of experienced burnout. Journal of Occupational Behaviour [Internet]. 1981 [consultado 2 de junio de 2016]; 2: 99-113. Disponible en: http://onlinelibrary.wiley.com/doi/10.1002/ job.4030020205/pdf

3. Vélez P, Mayorga LD. Relación de los factores de riesgo psicosociales y el síndrome de burnout en docentes de planta de la Universidad Francisco de Paula Santander en el periodo del 2011-2012. Rev. cienc. cuidad. [Internet]. 2013 [consultado 2 de junio de 2016]; 10(2); 85-95. Disponible en: http://revistas.ufps.edu.co/ojs/ index.php/cienciaycuidado/article/view/261/282

4. Quiceno JM, Vinaccia S. Burnout: Síndrome de quemarse en el trabajo (SQT). Acta Colombiana de Psicología [Internet]. 2007 [consultado 12 de marzo de 2015]; 10(2): 117-125. Disponible en: http://www.redalyc.org/ articulo.oa?id=79810212

5. Bareño J, Berbesi D, Montoya C. Factores asociados al Síndrome de burnout en docentes de enfermería MedellínColombia. INVESTIGACIONES ANDINA [Internet]. 2008 [consultado 16 de febrero de 2015]; 21(12):36-48. Disponible en: http://www.scielo.org.co/pdf/inan/v12n21/v12n21a04.pdf

6. Ortega $\mathrm{C}, \mathrm{López} \mathrm{F}$. El burnout o síndrome de estar quemado en los profesionales sanitarios: revisión y perspectivas. Inter Journ of Clinical and Health Psychology [Internet]. 2004 [consultado 16 de mayo de 2014]; 4(1): 137-160. Disponible en: http://www.aepc.es/ijchp/articulos_pdf/ijchp-100.pdf

7. Edmons C, Lockwood Gm, Bezjak A, Nyhof-Young J. Alleviating emotional exhaustion in oncology nurses: an evaluation of wellsprings "care for the professional caregiver program". J Cancer Educ. [Internet]. 2012 [consultado 13 de abril de 2015]; 27(1): 27-36 Disponible en: http://www.ncbi.nlm.nih.gov/pubmed/22042712

8. Kravits K, Mcallister-Blabck R, Grant M, Kirk C. Self-care strategies for nurses: a psycho- educational intervention for stress reduction and the prevention of SB. Applied Nursing Research [Internet]. 2010 [consultado 13 de abril de 2013]; 23(3): 130-138. Disponible en: http://www.ncbi.nlm.nih.gov/pubmed/20643322

9. Italia S, Favara C, Di Cataldo A, Russo G. Evaluation and art therapy treatment of the burnout syndrome in oncology units. Psycho-Oncology [Internet]. 2008 [consultado 12 de marzo de 2015]; 17 (7): 676-680. Disponible en: http://pesquisa.bvsalud.org/portal/resource/es/mdl-17992704

10. Tuesca R, Iguarán M, Suárez M, Vargas G, Vergara D. Síndrome de desgaste profesional en enfermeras/os del área metropolitana de Barranquilla. Salud Uninorte [Internet]. 2006 [consultado 26 de noviembre de 2014]; 22(2): 84-91. Disponible en: http://www.redalyc.org/pdf/817/81722204.pdf

11. Albaladejo R, Villanueva R, Ortega P, Astasio P, Calle Me, Domínguez V. Síndrome de burnout en el personal de enfermería de un hospital de Madrid. Rev. Esp. Salud Pública [Internet]. 2004 [consultado 6 de mayo de 2014]; 78(4): 505-516. Disponible en: http://www.scielosp.org/pdf/resp/v78n4/original3.pdf

12. Chacón M, Grau JA. Burnout y variables personales moduladoras en enfermeros que trabajan en hospitales oncológicos. Psicología y Salud [Internet].2004 [consultado 6 de junio de 2015]; 14(1):67-78. Disponible en: http://www.redalyc.org/pdf/291/29114108.pdf

13. Contreras FT, Juárez F, Murrain E. Influencia del Burnout, la calidad de vida y los factores socioeconómicos en las estrategias de afrontamiento utilizadas por los profesionales y auxiliares de enfermería. Pensamiento Psicológico [Internet]. 2008 [consultado 6 de mayo de 2014]; 4(11): 29-43. Disponible en: http://www.redalyc. org/articulo.oa?id $=80111671003$

14. Ourcilleón A, Abarca C, Puentes MV, Rodríguez P. Burnout en profesionales de enfermería de cuidados intensivos. Rev Chil Med Inten [Internet]. 2007 [consultado 12 de marzo de 2015]; 22(2): 83- 92. Disponible en: http://medicina-intensiva.cl/revistaweb/revistas/indice/2007-2/2.pdf

15. Vázquez J, Vázquez J, Álvarez M, Reyes M, Martínez R, Nadal M, et al. Intervención psicoterapéutica grupal intensiva realizada en un entorno natural para el tratamiento del burnout en un equipo de enfermería oncológica. Psicooncología [Internet]. 2012 [consultado 26 de noviembre de 2014]; 9(1):161-182. Disponible en: http:// revistas.ucm.es/index.php/PSIC/article/view/39145/37750

16. Edwards D, Burnard P. A systematic review of stress and stress management interventions for mental health nurses. J Adv Nurs. [Internet]. 2003 [consultado 3 de mayo de 2016]; 42 (2):169-200. Disponible en: http://www. ncbi.nlm.nih.gov/pubmed/12670386

17. Torres JM. Reflexiones sobre funciones del personal de enfermería. Rev Cubana Salud Pública [Internet]. 2004 [consultado 23 de noviembre de 2014]; 30(4): 0-0. Disponible en: http://scielo.sld.cu/scielo.php?pid=S0864- 
18. República de Colombia, Congreso de Colombia. Ley 266 de 1996, enero 25, por la cual se reglamenta la profesión de enfermería en Colombia y se dictan otras disposiciones. Diario Oficial No. 42.710. Bogotá: El Ministerio; 1996.

19. Guerrero J, Pulido G. Trabajo, salud y régimen contractual en personal de enfermería: un enfoque psicosocial. av. enferm. [Internet]. 2010 [consultado 16 de febrero de 2015]; 28(2): 111-122. Disponible en: http://www. revistas.unal.edu.co/index.php/avenferm/article/view/21386/22355

20. Melita A, Cruz M, Merino JM. Burnout en profesionales de enfermería que trabajan en centros asistenciales de la octava región, Chile. cienc. enferm. [Internet]. 2008 [consultado 2 de marzo de 2015]; XIV(2): 75-85. Disponible en: http://dx.doi.org/10.4067/S0717-95532008000200010

21. Aiken LH, Clarke SP, Sloane D, Sochalski JA, Busse R, Clarke H, et al. Nurses' reports on hospital care in five countries. Health Aff [Internet]. 2001 [consultado 29 de mayo de 2015]; 20(3):43-53. Disponible en: http:// content.healthaffairs.org/content/20/3/43. full

22. Grau A, Flichtentrei D, Suñer R, Prats M, Braga F. Influencia de factores personales, profesionales y transnacionales en el síndrome de burnout en personal sanitario hispanoamericano y español. Rev Esp Salud Pública [Internet]. 2009 [consultado 7 de junio de 2015]; 83(2): 215-230. Disponible en: http://scielo.isciii.es/ scielo.php?script $=$ sci arttext\&pid $=$ S1135-57272009000200006

23. Álvarez M, Lima H, Alfonso J, Torres A, Torres A. Síndrome de burnout y personal de enfermería de cuidados intensivos. Rev. Med. Electrón. [Internet]. 2009 [consultado 2 de marzo de 2015]; 31(3). Disponible en: http:// scielo.sld.cu/scielo.php?script $=$ sci arttext\&pid $=$ S1684-18242009000300009

24. Grisales H, Muñoz Y, Osorio D, Robles E. Síndrome de burnout en el personal de enfermería de un hospital de Ibagué, Colombia, 2014. Enfermería Global [Internet]. 2016 [consultado 7 de abril de 2016]; 41(1): 244-257. Disponible en: http://revistas.um.es/eglobal/article/viewFile/212851/188521

25. Cogollo Z, Batista E, Cantillo Ch, Jaramillo A, Rodelo D, Meriño G. Desgaste profesional y factores asociados en personal de enfermería de servicios de urgencias de Cartagena. Aquichán [Internet]. 2010 [consultado 2 de marzo de 2015]; 10(1): 43-51. Disponible en: http://aquichan.unisabana.edu.co/index.php/aquichan/article/ view/1605/2002

26. Ávila JH, Gómez LN, Montiel MM. Características demográficas y laborales asociadas al Síndrome de Burnout en profesionales de la salud. Pensamiento Psicológico [Internet]. 2010 [consultado 23 de noviembre de 2014]; 8(15):39-52. Disponible en: http://www.redalyc.org/pdf/801/80115648004.pdf

27. Laurenţiu P, Sava FA, Butta O. The effectiveness of controlled interventions on employees' burnout: A metaanalysis. Journal of Occupational and Organizational Psychology. [Internet]. 2016 [consultado 3 de mayo de 2016]; 89 (1): 1-27. Disponible en: http://onlinelibrary.wiley.com/doi/10.1111/joop.12099/abstract

28. Popp MS. Estudio preliminar sobre el síndrome de burnout y estrategias de afrontamiento en enfermeras de unidades de terapia intensiva (UTI). Interdisciplinaria [Internet]. 2008 [consultado 7 de junio de 2015]; 25(1):527. Disponible en: http://www.redalyc.org/articulo.oa?id=18025101

29. Günusën N, Üstün B. An RCT of coping an support groups to reduce burnout among nurses. Int Nurs Rev [Internet]. 2010 [consultado 16 de febrero de 2015]; 57(4):485-92. Disponible en: http://www.ncbi.nlm.nih.gov/ pubmed/21050201

30. Isaksson KE, Gude $\mathrm{T}$, Tyssen $\mathrm{R}$, Aasland OG. A self- referral preventive intervention for burnout amogn Norwegian nurses: one-year follow-up study. Patient Educ Couns [Internet]. 2010 [consultado 13 de abril de 2015]; 78(2): 191-7. Disponible en: http://www.ncbi.nlm.nih.gov/pubmed/19656650

31. Goodman MJ, Schorling JB. A mindfulness course decreases burnout and improves well-being among healtcare providers. Int J Psychiatry Med [Internet]. 2012 [consultado 13 de abril de 2015]; 43(2): 119-28. Disponible en: http://www.ncbi.nlm.nih.gov/pubmed/22849035

32. Sabanciogullari S, Dogan S. Effects of the professional identity development programme on the professional identity, job satisfaction and burnout levels of nurses: A pilot study. Int J Nurs Pract. [Internet]. 2015 [consultado enero de 2016]; 21(6): 847-57. Disponible en: http://www.ncbi.nlm.nih.gov/pubmed/24779558

33. Ewers P, Bradshaw T, Mcgovern J, Ewers B. Does training in psychosocial interventions reduce SB rates in forensic nurses?. Rev. Issues and Innovations in Nursing education. J Adv Nurs [Internet]. 2002 [consultado 3 de mayo de 2016]; 37(5): 470-6. Disponible en: http://www.ncbi.nlm.nih.gov/pubmed/11843986

34. Le Blanc P, Hox JJ, Schaufeli WB, Taris TW, Peeters MC. Take Care! The evaluation of a team-based burnout intervention program for oncology care providers. Journal of Applied Psychology [Internet]. 2007 [consultado 18 de mayo de 2016]; 92(1): 213-227. Disponible en: http://www.wilmarschaufeli.nl/publications/Schaufeli/263. pdf 
ISSN 1794-9831

E-ISSN 2322-7028 Vol. 14 No. 1 Ene - Jun 2017 Cúcuta, Colombia
35. Quenot JP, Rigaud JP, Prin S, Barbar S, Pavon A, Hamet M, et al. Suffering among carers working in critical care can be reduced by an intensive communication strategy on end-of-life practices. Intensive Care Medicine [Internet]. 2012 [consultado 25 de abril de 2015]; 38(1): 55-61. Disponible en: http://www.ncbi.nlm.nih.gov/ pubmed/22127481

36. Rodrigues A, Carneseca EC, Paiva CE, Ribeiro BS. Impacto de un programa de actividad física sobre la ansiedad, la depresión, el estrés ocupacional, y en síndrome de agotamiento en el trabajo de los profesionales de enfermería. Rev Latino-Am.Enfermagen [Internet]. 2014 [consultado 2 de marzo de 2015]; 22(2): 332-6. Disponible en: http://www.scielo.br/pdf/rlae/v22n2/es 0104-1169-rlae-22-02-00332

37. Günusën N, Üstün B. Turkish nurses' perspectives on a programme to reduce burnout. Int NursRev [Internet]. 2009 [consultado 16 de febrero de 2015]; 56 (2): 237-42. Disponible en: http://www.ncbi.nlm.nih.gov/ pubmed/19646174

38. Bueno LE, Santamaría NP. Acciones para mejorar el estrés laboral de las enfermeras en servicios de oncología. Revisión documental de 1995 a 2005. Rev Med UNAB [Internet]. 2008 [consultado 25 de mayo de 2016]; 11 (2):154-162. Disponible en: http://revistas.unab.edu.co/index.php?journal=medunab\&page=article\&op=view\& path $\% 5 \mathrm{~B} \% 5 \mathrm{D}=76 \&$ path $\% 5 \mathrm{~B} \% 5 \mathrm{D}=69$

39. Thofehm MB, López-Montesinos MJ, Amestoy SC, Porto AR, Bettin A, Fernandes HN, et al. Trabajo en equipo: visión de los enfermeros de un hospital de Murcia/España. Enferm. glob. [Internet]. 2014 [consultado 7 de junio de 2015]; 13(36): 223-237. Disponible en: http://revistas.um.es/eglobal/article/view/190421/165721

40. Montoya PA, Moreno S. Relación entre síndrome de burnout, estrategias de afrontamiento y engagement. Psicología desde el Caribe [Internet]. 2012 [consultado 3 de marzo de 2015]; 29(1): 205-227. Disponible en: http://rcientificas.uninorte.edu.co/index.php/psicologia/article/view/1940/3905

41. Berrios MP, Augusto JM, Aguilar MC. Inteligencia emocional percibida y satisfacción laboral en contextos hospitalarios: Un estudio exploratorio con profesionales de enfermería. Index Enferm [Internet]. 2006 [consultado 8 de junio de 2015]; 15(54): 30-34. Disponible en: http://scielo.isciii.es/scielo.php?script=sci_artte xt\&pid=S1132-12962006002200006

42. Davis S, Lind BK, Sorensen C. A Comparison of SB Among Oncology Nurses Working in Adult and Pediatric Inpatient and Outpatient Settings. Oncol Nurs Forum [Internet]. 2013 [consultado 25 de abril de 2015]; 40(4): 303-11. Disponible en: http://www.ncbi.nlm.nih.gov/pubmed/23803274

43. Beltrán C, Pando M, Pérez MB. Apoyo social y síndrome de quemarse en el trabajo o bournout: una revisión psicología y salud. Psicología y Salud [Internet]. 2004 [consultado 3 de marzo de 2015]; 4 (1): 79-87: Disponible en: http://www.redalyc.org/pdf/291/29114109.pdf

44. Damiani JS. Habilidades comunicativas autovaloradas en profesores de ciencias básicas biomédicas. Rev haban cienc méd [Internet]. 2011 [consultado 8 de junio de 2015]; 10(4): 436-440. Disponible en: http://scielo.sld.cu/ scielo.php?script $=$ sci_arttext\&pid $=$ S1729-519X2011000400004

45. Vega OM, González DS. Apoyo social: elemento clave en el afrontamiento de la enfermedad crónica. Enferm. glob. [Internet]. 2009 [consultado 8 de junio de 2015]; 8(2). Disponible en: http://revistas.um.es/eglobal/article/ view/66351/63971

46. Albarrán AJ, Macías MA. Aportaciones para un modelo psicoeducativo en el servicio de psiquiatría del Hospital Civil Fray Antonio Alcalde en Guadalajara, Jalisco, México. Investigación en Salud [Internet]. 2007 [consultado 3 de junio de 2015]; IX(2): 118-124. Disponible en: http://www.redalyc.org/articulo.oa?id=14290206

47. León CA. Cuidarse para no morir cuidando. Rev Cubana Enfermer [Internet]. 2007 [consultado 8 de junio de 2015]; 23(1): 1-9. Disponible en: http://www.bvs.sld.cu/revistas/enf/vol23 01 07/enf06107.htm

48. Chang Y, Chan HJ. Optimism and proactive coping in relation to burnout among nurses. J Nurs Manag. [Internet]. 2015 [consultado 23 de mayo de 2016]; 23(3): 401-8. Disponible en: http://www.ncbi.nlm.nih.gov/ pubmed/24112222

49. Espina JA. Integración del psicodrama con otras líneas teóricas. Rev. Asoc. Esp. Neuropsiq [Internet]. 2001 [consultado 7 de junio de 2015]; (77): 33-49. Disponible en: http://www.revistaaen.es/index.php/aen/article/ view/15745/15604

50. Terroni NN. La comunicación y la asertividad del discurso durante las interacciones grupales presenciales y por computadora. Psico-USF [Internet]. 2009 [consultado 6 de junio de 2015]; 14 (1): 35-46. Disponible en: http:// www.scielo.br/pdf/pusf/v14n1/a05v14n1.pdf

51. Görgens G, Brand T. Emotional intelligence as a moderator in the stress-burnout relationship: a questionnaire study on nurses. J Clin Nurs [Internet]. 2012 [consultado 12 de abril de 2015]; 21(16-16): 2275-85. Disponible en: http://www.ncbi.nlm.nih.gov/pubmed/22788561

52. Undurraga DG, Martínez XF. "burnout" o agotamiento en la práctica diaria de medicina. Rev Chil Cardiol 
[Internet]. 2009 [consultado 8 de junio de 2015]; 28: 403-307. Disponible en: http://www.scielo.cl/pdf/rchcardiol/ v28n4/art13.pdf

53. Da Silva MA, Braga M, Da Silva CT. Evaluación de la presencia del síndrome de bournout en cuidadores de ancianos. Enferm. glob. [Internet]. 2009 [consultado 9 de junio de 2015]; 16(2). Disponible en: http://revistas. um.es/eglobal/article/view/65451/65721

54. Taormina RJ, Law CM. Approaches to preventing burnout: the effects of personal stress management and organizational socialization. J Nurs Manag [Internet]. 2000 [consultado 19 de mayo de 2016]; 8(2): 89-99. Disponible en: http://www.ncbi.nlm.nih.gov/pubmed/11051965
ISSN 1794-9831

E-ISSN 2322-7028

Vol. 14 No. 1

Ene - Jun 2017

Cúcuta, Colombia 